\title{
The Derivation of Direction Selectivity in the Striate Cortex
}

\author{
Matthew R. Peterson, Baowang Li, and Ralph D. Freeman \\ Group in Vision Science, School of Optometry, Helen Wills Neuroscience Institute, University of California, Berkeley, Berkeley, California 94720-2020
}

In the central visual pathway of binocular animals, the property of directional selectivity (DS) is first exhibited in striate cortex. In this study, we sought to determine the neural circuitry underlying the transformation from non-DS neurons to DS cortical cells. In a well established model, DS receptive fields (RFs) are derived from the sum of two non-DS inputs with $90^{\circ}$ (quadrature) spatiotemporal phase differences. We explored possible input sources for this model, which include non-DS simple cells and lateral geniculate nucleus (LGN) neurons, by examination of spatiotemporal RFs of single cells and of pairs of cells. We find that distributions of non-DS simple RFs do not match the temporal predictions of the quadrature model because of a lack of long-latency responses. The long-latency inputs could potentially arise from lagged LGN afferents. However, analysis of cell pairs indicates that DS cells receive cortical input from non-DS simple cells for both short- and long-latency components, with temporal phase differences typically $<90^{\circ}$. Furthermore, the distribution of minimum phase differences needed to generate DS cells overlaps that exhibited by non-DS simple cells. Considered together, these results are consistent with a linear model whereby DS simple cells are formed from simple-cell inputs, with temporal phase differences often less than quadrature.

Key words: directional; extracellular; lateral geniculate; LGN; receptive field; striate cortex; vision

\section{Introduction}

Determination of the direction of stimulus motion is a fundamental encoding requirement of the visual system. This is accomplished by retinal ganglion cells in animals with minimal binocular field overlap (Barlow and Levick, 1965; Borg-Graham, 2001; Taylor and Vaney, 2003). In species with frontally positioned eyes, directional selectivity (DS) is a cortical function (Hubel and Wiesel, 1962). A central question of visual processing concerns the determination of how non-DS input generates DS simple cells in the striate cortex. To address this question, it is useful to consider the energy model (Adelson and Bergen, 1985; Watson and Ahumada, 1985), which provides a basis for the derivation of direction selectivity. According to this theoretical construct, a pair of input filters with quadrature spatial and temporal phase differences can generate a DS receptive field (RF). This model has been used effectively in previous work (DeAngelis et al., 1993; Jagadeesh et al., 1997; De Valois and Cottaris, 1998; De Valois et al., 2000). For the current purpose, it is necessary to consider what inputs are physiologically plausible.

A recent examination of RFs in the monkey visual cortex (De Valois and Cottaris, 1998; De Valois et al., 2000) suggests the existence of two subpopulations of non-DS simple cells with the appropriate phase relationships to serve as inputs for the quadrature model. One population exhibits biphasic temporal response profiles with a short-latency onset (early phase), and the other has

\footnotetext{
Received Dec. 6, 2003; revised Feb. 27, 2004; accepted Feb. 27, 2004.

This work was supported by National Eye Institute Research and Core Grants EY-01175 and EY-03176. We thank G. DeAngelis for helpful comments on a draft of this manuscript.

Correspondence should be addressed to Ralph D. Freeman, Group in Vision Science, School of Optometry, Helen Wills Neuroscience Institute, University of California, Berkeley, 360 Minor Hall, Berkeley, CA 94720-2020. E-mail: freeman@neurovision.berkeley.edu.

DOI:10.1523/JNEUROSCI.5398-03.2004

Copyright $\odot 2004$ Society for Neuroscience $\quad$ 0270-6474/04/243583-09\$15.00/0
}

monophasic responses with longer latencies (late phase). However, subpopulations of simple cells with this quadrature relationship do not appear to be present in the cat because of a lack of neurons with the appropriate late-phase response profiles (Dean and Tolhurst, 1986; DeAngelis et al., 1999; Nishimoto et al., 2001). Some studies have proposed that DS simple cells in the cat receive late-phase inputs directly from lagged lateral geniculate nucleus (LGN) afferents, either in combination with other LGN cells (Saul and Humphrey, 1990) or with non-DS simple cells (Nishimoto et al., 2001).

Simple cells exhibit a broad distribution of selectivity to direction (Schiller et al., 1976; Hamilton et al., 1989; Gizzi et al., 1990; De Valois et al., 2000). In the quadrature model, different degrees of selectivity are generated by weighted sums of the input components. Strongly and weakly selective units are created by balanced and unbalanced weights, respectively. In an alternative scheme, which we refer to as the "variable phase model," different degrees of selectivity are obtained by summation with balanced weights and phase differences in the range of $0-90^{\circ}$.

In the study reported here, we assess the roles of non-DS simple cells and LGN input in the generation of DS simple cells in area 17 of the cat. We determined the temporal input requirements of a large sample of DS cells according to the quadrature and variable phase models and compare these requirements with the temporal characteristics of non-DS simple and LGN RFs. More direct evaluations of intracortical connections are made by analysis of RFs of pairs of simultaneously recorded simple cells. Our results are consistent with a variable phase model with simple-cell inputs.

\section{Materials and Methods}

Extracellular recordings are made from cells in area 17 and LGN of anesthetized (sodium thiamylal or thiopental administered at a rate deter- 
mined individually for each animal) and paralyzed (gallamine triethiodide, $10 \mathrm{mg} \cdot \mathrm{kg}^{-1} \cdot \mathrm{hr}^{-1}$, or pancuronium, $0.1-0.2 \mathrm{mg} \cdot \mathrm{kg}^{-1} \cdot \mathrm{hr}^{-1}$ ) adult cats. All procedures were conducted in conformance with the guidelines regarding the care and use of animals adopted by the Society for Neuroscience and according to local campus regulations. Single-unit recordings are obtained using multiple (two to four) tungsten microelectrodes (Levick, 1972; or commercial models) with impedances between 2 and $10 \mathrm{M} \Omega$. For area 17 recordings, electrode penetrations are made down the medial bank of the postlateral gyrus at P4 L2.5 in HorsleyClarke (H-C) coordinates (Horsley and Clarke, 1908) angled $10^{\circ}$ medial and $20^{\circ}$ anterior. Vertical electrode penetrations are made for LGN recordings at $\mathrm{H}-\mathrm{C}$ coordinates A6 L9. After a unit is identified by its response waveform, RF parameters are measured using drifting sinusoidal gratings and random-noise stimuli. Details of the surgical and experimental procedures have been described previously (Cai et al., 1997; Anzai et al., 1999).

Cell classification. Cortical cells are classified as simple or complex by classical criteria (Hubel and Wiesel, 1962) and by comparing the first harmonic (F1) to the DC (F0) of the peristimulus time histogram (PSTH) to a grating drifting at $2 \mathrm{~Hz}$ with optimized spatial frequency and orientation. Cells with an F1/F0 ratio $\geq 1$ are classified as simple (Skottun et al., 1991) (for an alternative view of this classification system, see Mechler and Ringach, 2002). In the LGN, X and Y cells exhibit similar linear RF organization (Cai et al., 1997), and we have not differentiated them in this study. W cells located in the C laminas (Cleland et al., 1976; Wilson et al., 1976) have been avoided. LGN cells are classified as lagged or nonlagged (Mastronarde, 1987; Saul and Humphrey, 1990) on the basis of the absolute value of the ratio of the first to second peak amplitudes of the temporal profile of the RF center. Values $<1$ are classified as lagged, otherwise nonlagged (Cai et al., 1997; Wolfe and Palmer, 1998). Spatiotemporal RFs are measured using a reverse correlation method (see below). This classification system is simpler than the set of tests delineated by Mastronarde (1987) and is similar to the rebound index used by Alonso et al. (2001).

Grating measurements. Spatial and temporal tuning parameters are measured using drifting sinusoidal gratings. Gratings are presented monocularly at $50 \%$ Michelson contrast with a mean luminance of $17 \mathrm{~cd} /$ $\mathrm{m}^{2}$. For orientation, direction selectivity, spatial frequency, and size tuning measurements, gratings are drifted at a temporal frequency of $2 \mathrm{~Hz}$ with other nonvariable parameters optimized for the cell. For temporal frequency tuning measurements, optimal size, spatial frequency, and orientation parameters are used. For direction selectivity measurements, the F1 amplitude of the PSTH is obtained in response to the preferred (P) and nonpreferred (NP) directions to calculate a direction selectivity in$\operatorname{dex}(\mathrm{DSI})$ :

$$
\text { DSI }=1-\frac{\mathrm{NP}}{\mathrm{P}}
$$

RF measurements. Estimates of linear spatiotemporal RFs are measured using a reverse correlation procedure (Jones and Palmer, 1987; Freeman and Ohzawa, 1990). For most cells, a spatially one-dimensional spacetime RF is obtained using long bars oriented along the preferred orientation of the cell. For a subpopulation of cells, a more time consuming two-dimensional RF is measured. For in-depth descriptions of the reverse correlation procedures as used here, see DeAngelis et al. (1993). Briefly, individual bar stimuli of either moderate $\left(32 \mathrm{~cd} / \mathrm{m}^{2}\right)$ or low $(2$ $\mathrm{cd} / \mathrm{m}^{2}$ ) luminance are displayed one at a time on random grid locations for $40 \mathrm{msec}$ durations with a mean background luminance of $17 \mathrm{~cd} / \mathrm{m}^{2}$. By cross-correlating the stimulus with the response, a linear approximation to the space-time RF is obtained. Before spatial and temporal parameters are computed, the RF is first interpolated using a cubic spline.

Predicted direction selectivity. The direction selectivity predicted from the linear space-time RF is determined by calculating the spatiotemporal amplitude spectrum by Fourier analysis. The predicted responses to a grating drifting in the preferred and nonpreferred directions at a given spatial frequency and $2 \mathrm{~Hz}$ temporal frequency are obtained by finding the values of the amplitude spectrum at that spatial frequency for +2 and $-2 \mathrm{~Hz}$ temporal frequencies, respectively (DeAngelis et al., 1993). A DSI value is computed from the predicted responses using Equation 1.
Receptive field fitting. Spatiotemporal RFs, $R(x, t)$, are fit using a sum of two space-time separable components:

$$
R(x, t)=G(x) H(t)+G^{\prime}(x) H^{\prime}(t)
$$

where $G(x) H(t)$ and $G^{\prime}(x) H^{\prime}(t)$ are the two components. Here, we model the spatial profile, $G(x)$, as a Gabor function (Gabor, 1946):

$$
G(x)=e^{\left.-2\left(x-x_{0}\right) / w\right]^{2}} \cos \left(2 \pi f_{x}\left(x-x_{0}\right)+\phi_{x}\right),
$$

which provides a good fit for simple-cell RFs (Field and Tolhurst, 1986; Jones and Palmer, 1987; DeAngelis et al., 1993). In general, the temporal profile, $H(t)$, is not as well described by a pure Gabor function. The typical temporal response of a simple cell exhibits a rapid onset and a gradual offset and is better fit by a sigmoidally skewed Gabor function (DeAngelis et al., 1999):

$$
\begin{gathered}
G_{t}(t)=e^{-2\left[\left(t-t_{0}\right) / c\right]^{2}} \cos \left(2 \pi f_{t}\left(t-t_{0}\right)+\phi_{t}\right), \\
T(t)=\frac{2}{\pi} \tan ^{-1}(\beta t), \\
H(t)=G_{t}(T(t)),
\end{gathered}
$$

where $T(t)$ is the temporal skewing function.

The second separable component, $G^{\prime}(x) H^{\prime}(t)$, is modeled with the same parameters as the first but with phase offsets in both space,

$$
G^{\prime}(x)=e^{-2\left[\left(x-x_{0}\right) / w\right]^{2}} \cos \left(2 \pi f_{x}\left(x-x_{0}\right)+\phi_{x}+\Delta \phi_{x}\right),
$$

and time,

$$
\begin{gathered}
G^{\prime}{ }_{t}(t)=e^{-2\left[\left(t-t_{0}\right) / c\right]^{2}} \cos \left(2 \pi f_{t}\left(t-t_{0}\right)+\phi_{t}+\Delta \phi_{t}\right), \\
H^{\prime}(t)=G^{\prime}{ }_{t}(T(t)) .
\end{gathered}
$$

For a quadrature fit to a DS (i.e., space-time inseparable) RF, the special and temporal offsets $\left(\Delta \varphi_{x}\right.$ and $\left.\Delta \varphi_{t}\right)$ are both fixed at $90^{\circ}$. Different levels of direction selectivity are obtained by summing the two components with different relative amplitudes:

$$
R_{\text {quad }}(x, t)=k\left[G(x) H(t)+\alpha G^{\prime}(x) H^{\prime}(t)\right],\left\{\Delta \phi_{x}=\Delta \phi_{t}=90^{\circ}\right\},
$$

where the resulting RFs vary from non-DS to maximally DS as $\alpha$ varies from 0 to 1 . The $k$ parameter scales the overall amplitude.

For a variable phase fit, different degrees of direction selectivity are obtained by summing the two components with equal amplitudes (i.e., $\alpha=1)$ and allowing $\Delta \varphi_{x}$ and $\Delta \varphi_{t}$ to vary:

$$
R_{\text {nonquad }}(x, t)=k\left[G(x) H(t)+G^{\prime}(x) H^{\prime}(t)\right],\left\{-90^{\circ} \leq \Delta \phi_{x, t} \leq 90^{\circ}\right\} .
$$

The actual resulting temporal phase difference between the two components is a function of, but not always equal to, $\Delta \varphi_{t}$. The inequality is a result of the Gaussian envelope and temporal skew. For this reason, reported phase differences are determined by Fourier analysis, in which the characteristic phase of each component is defined at the peak temporal frequency for the inseparable RF.

Cell pair analysis. We record simultaneously a pair of RFs from potentially connected simple cells, in which one is DS and the other non-DS. We define "potentially connected" as having overlapping spatial RFs ( $>50 \%$ overlap) with similar orientation and spatial frequency preferences. Such pairs of cells have a high probability of being functionally connected (Ghose et al., 1994). More direct estimates of the underlying neural connections are obtained by cross-correlation (see below). However, because of the difficulties in interpreting cross-correlation histograms (CCHs) (Brody, 1998, 1999), especially with relatively low neural response rates, the classification of potentially connected is not dependent on this analysis. Here we assume that the non-DS cell of each potentially connected pair forms one of the input components to the DS cell. This allows us to infer properties of the missing input component. 


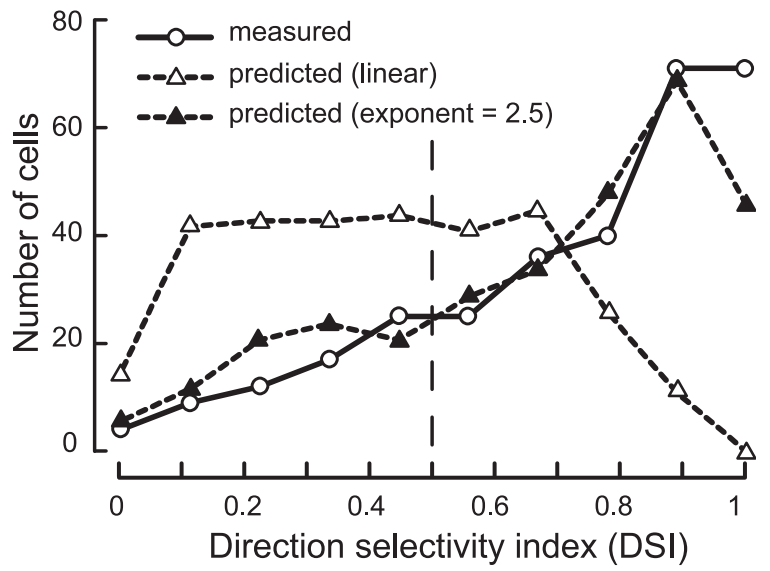

Figure 1. Different measurements of the distribution of DSI for a sample of simple cells ( $n=$ 310). Open circles, The distribution of DSI measured using drifting gratings. Open triangles, The distribution of DSI predicted from the linear RF. Filled triangles, The distribution of DSI predicted from the RF taking into account an expansive output nonlinearity modeled as an exponent of 2.5. In addition to having a measured DSI $>0.5$, cells must also have a predicted DSI $>0.5$ (as indicated by the vertical dashed line) to be classified as a DS cell.

To estimate the shape of the RF of the missing component, we first fit the measured DS and non-DS RFs with inseparable (variable $\alpha$ ) and separable $(\alpha=0)$ versions of the quadrature fitting function (Eq. 10), respectively. Then the "missing component" is obtained by fitting the difference of the pair of RF fits with a separable function $(\alpha=0$; Eq. 10). To be included in the pairs analysis, the sum of the missing component and the non-DS RF must account for at least $66 \%$ of the variance of the DS RF.

An estimate of the synaptic connectivity between two simultaneously recorded cells is obtained via averaged cross-correlation of spiking responses across multiple visual stimulus presentations. Artifacts in the resulting $\mathrm{CCH}$ attributable to stimulus correlations are corrected by a shuffled subtraction technique (Perkel et al., 1967). The type of functional connectivity between the two cells is inferred from the shape of the corrected CCH (Gerstein and Perkel, 1969; Toyama et al., 1981; Ghose et al., 1994; Menz and Freeman, 2004). Here we define a sharp peak in the $\mathrm{CCH}$ (width $<1 \mathrm{msec}$ ) displaced from zero by $<3 \mathrm{msec}$ as evidence of a monosynaptic excitatory connection. Broad peaks centered at zero are classified as common input.

\section{Results}

Distribution of degrees of direction selectivity

We measured space-time RFs for 310 simple cells from area 17 of the cat. We divided this sample into DS and non-DS units on the basis of the DSI of the cell determined both with drifting gratings and from RF analysis. The distribution of DSI values measured with drifting gratings is shown in Figure 1 (open circles). A value of 0.5 provides a good division between cells with RFs that appear separable and those that seem inseparable by visual inspection. However, a small number of cells with DSIs $>0.5$ exhibited space-time RFs that appeared separable. Because of this, the DSI predicted from the space-time RF was also taken into account when classifying a cell as DS. Figure 1 also shows the distribution of DSI values predicted from the linear RF (open triangles). This distribution is similar to that obtained from membrane potential recordings (Jagadeesh et al., 1997). The difference in the shape of the distribution arises because of nonlinearities in the transformation of membrane potentials to spiking activity, which is well modeled as a static power function with a mean exponent of 2.5 (DeAngelis et al., 1993). This expansive output nonlinearity enhances the direction selectivity measured from spiking responses, which shifts the DSI distribution to higher values. When an exponent of 2.5 is applied to the responses predicted from the linear

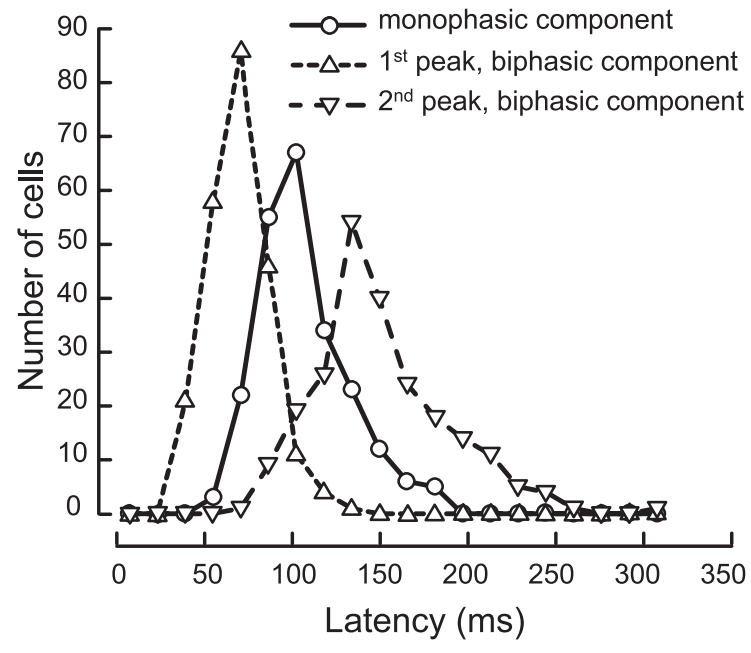

Figure 2. Distribution of latency-to-peak for quadrature fitting components of DS cells. The solid line shows time-to-peak latencies for monophasic fitting components (mean, $106.6 \pm$ $25.5 \mathrm{msec}$ ). The short and long dashed lines show the latencies for the first (upward triangles; mean, $69.1 \pm 17.3 \mathrm{msec}$ ) and second (downward triangles; mean, $150.0 \pm 37.8 \mathrm{msec}$ ) peaks of the biphasic fitting components, respectively.

RFs, the resulting DSI distribution (Fig. 1, filled triangles) is remarkably similar to the measured one (Fig. 1, open circles). Here we define cells as DS only when both measured and predicted (exponent, 2.5) DSI values are $>0.5$. Otherwise, cells are defined as non-DS. We should note that, in our sample, all cells with predicted DSIs $>0.5$ also had measured DSIs $>0.5$. Most cells in our sample (74\%) are classified as DS using these criteria.

\section{Temporal requirements of the quadrature model}

To estimate the temporal requirements of the quadrature model, we fit DS RFs with a quadrature-pair function (Eq. 10). We note that DS RFs tend to exhibit similar fitting components. For each cell, one of the components can be characterized by a fast biphasic temporal response and the other by a lagged monophasic temporal response. This is similar to the principal components found for DS RFs in the monkey (De Valois et al., 2000) and the cat (Nishimoto et al., 2001). The peak in the distribution of latencies for monophasic components (Fig. 2, open circles, solid line) falls approximately halfway between the latencies of the first and second peaks of biphasic components (short and long dashed lines), indicating a quadrature relationship. The second peak of the biphasic components exhibits a broader latency distribution (SD, 38 ) than the first $(\mathrm{SD}, 17)$, consistent with previous findings (De Valois et al., 2000) and partially reflecting the range of temporal frequency preferences for DS cells.

\section{Temporal characteristics of non-DS simple cells}

The latency distributions shown in Figure 2 represent estimates of the temporal requirements for inputs to DS cells based on the quadrature model. There are several input sources that could plausibly meet these requirements. Here, we considered direct input from LGN and from non-DS simple cells. In the monkey, there is evidence for two subpopulations of non-DS simple cells exhibiting monophasic and biphasic temporal responses, with phase differences in agreement with the quadrature model (De Valois et al., 2000). Some studies have suggested that similar subpopulations may not be present in the cat (DeAngelis et al., 1999; Nishimoto et al., 2001). To explore this, we divided non-DS cells into monophasic and biphasic subpopulations by use of an 


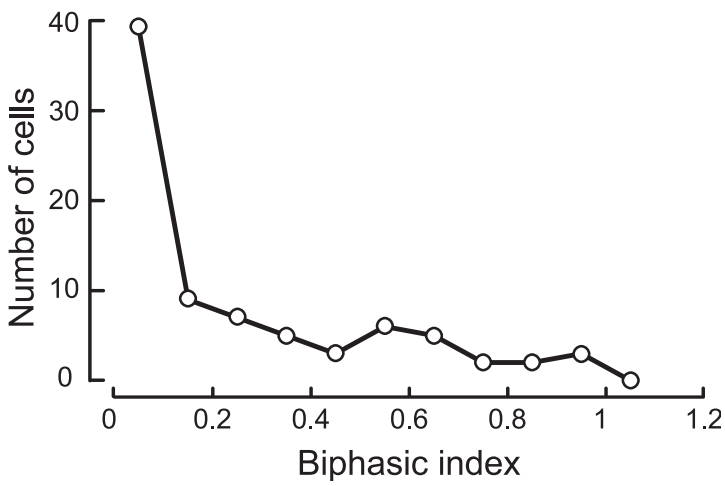

Figure 3. Distribution of biphasic indices for non-DS simple cells. Cells with biphasic indices $<0.3$ are classified as monophasic. Cells with biphasic indices $>0.5$ are classified as biphasic.

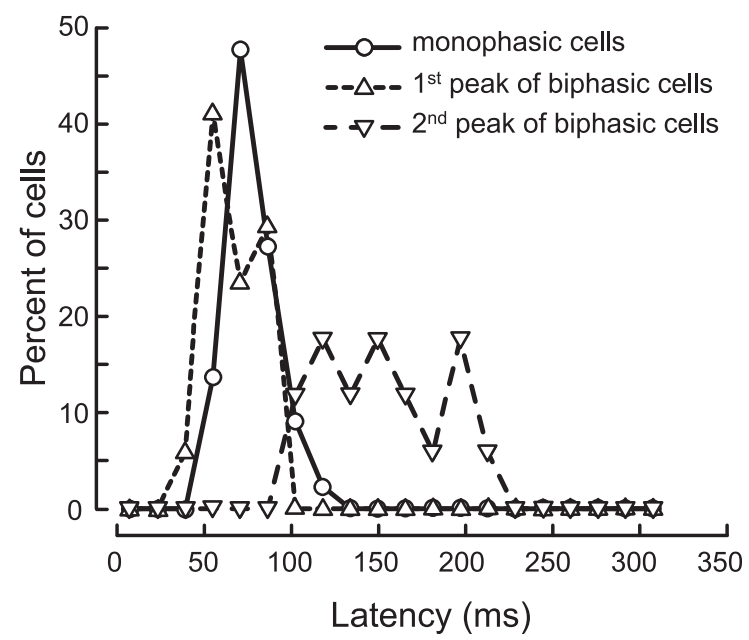

Figure 4. Distribution of latency-to-peak for non-DS simple cells. The solid line shows timeto-peak latencies for monophasic cells (mean, $76.3 \pm 14.4 \mathrm{msec}$ ). The short and long dashed lines show the latencies for the first (mean, $65.3 \pm 15.5 \mathrm{msec}$ ) and second (mean, $152.8 \pm 36.4$ msec) peaks of biphasic cells, respectively. The latencies of monophasic cells and those of the first peak of biphasic cells primarily overlap and show only weakly significant differences ( $p<$ $0.05 ;$ t test)

index consisting of the ratio of first to second peak amplitudes along the optimal time slice (i.e., biphasic index). Values near 1 indicate temporally biphasic responses with similar peak amplitudes, whereas those near 0 represent monophasic profiles. The distribution of biphasic indices shown in Figure 3 is similar to that found for the monkey (De Valois et al., 2000). Using the same conventions as De Valois et al. (2000), we classify cells with indices $>0.5$ as biphasic and those $<0.3$ as monophasic. The eight cells with indices between these values are unclassified and have been removed from the analysis.

Biphasic cells (Fig. 4, short and long dashed lines) exhibit temporal characteristics similar to the biphasic fitting components (Fig. 2, short and long dashed lines). The first and second peaks of the biphasic cells exhibit mean latencies of $65 \pm 16$ and $152 \pm 36 \mathrm{msec}$, respectively. These latencies may be compared with $69 \pm 17$ and $150 \pm 37 \mathrm{msec}$ for the first and second peaks of the biphasic fitting components. These are consistent with other findings that suggest that biphasic simple cells exhibit the latencies expected from the early component of the quadrature model (Nishimoto et al., 2001).

As a population, monophasic simple cells exhibit only slightly longer latencies $(76 \pm 14 \mathrm{msec}$ ) (Fig. 4, solid line) than the first

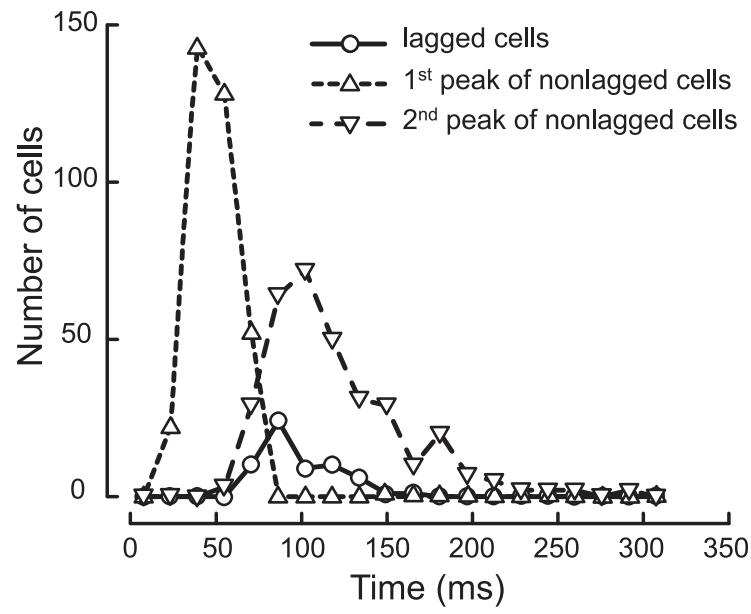

Figure 5. The distribution of peak latencies for different classes of LGN cells. The solid line shows the latency-to-peak for lagged cells ( $n=61$; mean, $97.5 \pm 22.2 \mathrm{msec}$ ). Short and long dashed lines show, respectively, first (mean, $49.4 \pm 12.0 \mathrm{msec}$ ) and second (mean, $119.8 \pm$ $40.2 \mathrm{msec}$ ) peak latencies for nonlagged cells $(n=345)$.

peak of biphasic cells $(65 \pm 16 \mathrm{msec})$ (Fig. 4 , short dashed line) and not as much as expected by the quadrature model $(107 \pm 26$ msec) (Fig. 2, solid line). The latency distribution of monophasic cells does not change significantly even if we narrow our definition to biphasic indices $<0.2$ (data not shown). This indicates that the temporal characteristics of non-DS simple cells in the cat only agree with the early component of the quadrature model, implying a possible role for direct input from lagged LGN cells (Nishimoto et al., 2001). This also suggests a possible species difference with the monkey in which population latency distributions for monophasic and biphasic simple cells form an approximately quadrature relationship (De Valois et al., 2000; Conway and Livingstone, 2003).

\section{Temporal characteristics of LGN cells}

One possibility is that the longer-latency components that feed into DS simple cells of the cat come directly from lagged LGN afferents (Saul and Humphrey, 1990; Nishimoto et al., 2001). To explore this potential mechanism, we analyzed the spatiotemporal RFs of 406 LGN cells. We recorded from 345 nonlagged and 61 lagged cells. In other words, we classified $15 \%$ of LGN cells as lagged. Other estimates of the percentage of lagged cells in the cat LGN range from $<20 \%$ (Kwon et al., 1992; Alonso et al., 2001) to $\sim 40 \%$ (Mastronarde, 1987; Humphrey and Weller, 1988). The broad range of estimates has been attributed to differences in classification criteria and the impedance properties of recording electrodes (Saul and Humphrey, 1992). Despite a likely under sampling, the lagged cells we find appear to have the desired latency requirements for late components of the quadrature model (Fig. 5, solid line). The mean latency of lagged cells (98 \pm $22 \mathrm{msec}$ ) is just slightly less than that of the monophasic fitting components (107 $\pm 25 \mathrm{msec}$ ), as is expected given thalamocortical conduction delays of a few milliseconds (Hawken et al., 1996). Assuming a 3 msec delay, the latencies of lagged cells are not statistically different from those of the monophasic fitting components ( $p>0.05 ; t$ test). However, the latencies of the first peak of nonlagged LGN cells $(49 \pm 12 \mathrm{msec}$ ) are substantially faster than those of the biphasic fitting components $(69 \pm 17$ $\mathrm{msec})$, even after accounting for a thalamocortical time delay $(p<0.01 ; t$ test). Also note that the relationship between lagged and nonlagged latency distributions resembles more of a $180^{\circ}$ 
A

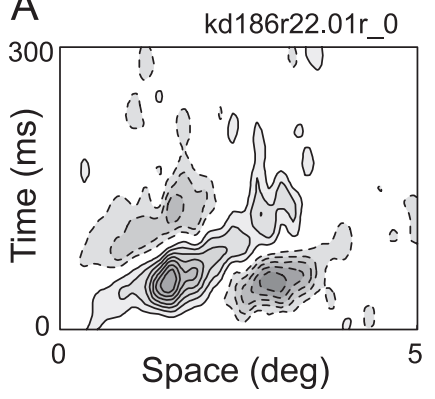

B

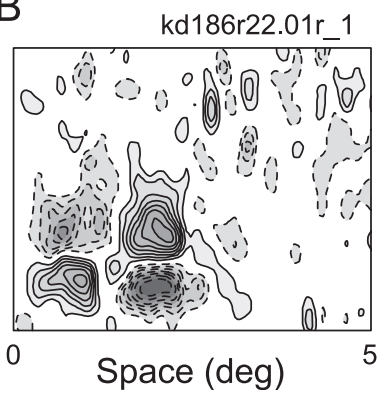

C

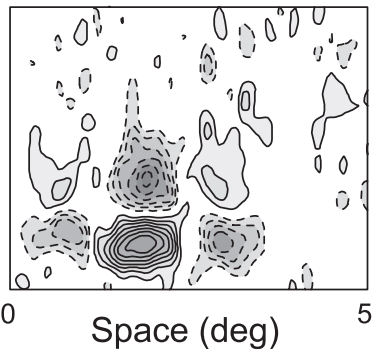

Figure 6. The difference between space-time RFs for a pair of potentially connected simple cells. $A$, The RF for the DS member of the cell pair. Solid and dashed contours represent "on" and "off" responses, respectively. $B$, The RF for the non-DS member of the cell pair. C, The space-time RF obtained by taking the difference of the DS and non-DS RFs. Here the difference resembles a separable (non-DS) cell, which could potentially be a simple cell, or a group of LGN cells.
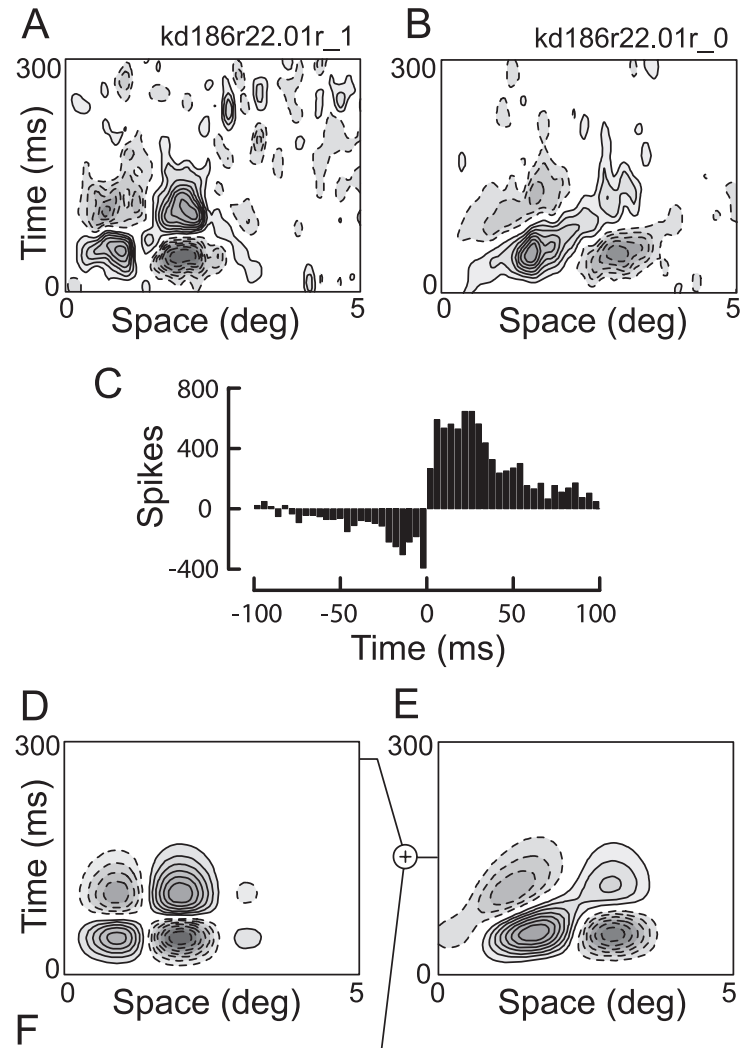

$\mathrm{E}$
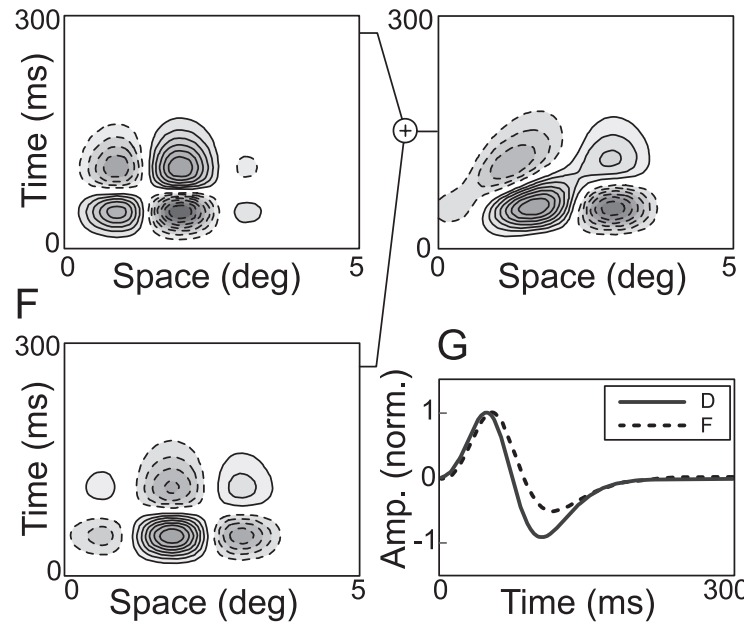

G

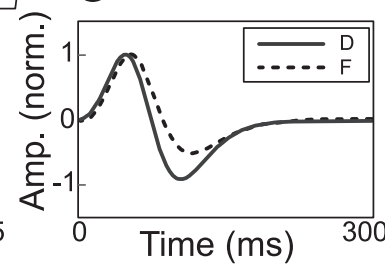

Figure 7. Example pair analysis for the cells shown in Figure 6.A,B, The non-DS and DS RFs. C, The CCH of the DS and non-DS cells. This CCH does not show a clear functional connection. The horizontal axis of the $\mathrm{C} C \mathrm{H}$ has been expanded to show the slow (not functionally significant) correlations. For this analysis, we assume the non-DS cell is one of two inputs to the DS cell. The analysis shown here is designed to determine the RF of the best-fitting second input. $D-F$, The fit to the second, i.e., missing, component $(F)$, which when added to the fit of the non-DSRF $(D)$, provides the best fit $\left(E ; R^{2}=0.88\right)$ to the $D S$ member of the pair $(B) . G$, The temporal profiles of the two input RFs. Solid and dashed lines represent the temporal profiles obtained from the components in $D$ and $F$, respectively. Here the temporal phase difference between the two components is only $11^{\circ}$.

phase difference than a quadrature relationship. In other words, the latencies of the lagged peaks fall within the latency distribution of nonlagged second peaks. A similar observation has been made previously (Alonso et al., 2001).

On the basis of temporal requirements of the quadrature model, which we estimate from a quadrature fit to DS simple cells, a combination of biphasic simple cells and lagged LGN cells appears to provide the required circuit. However, this nonhierarchical model implies an elaborate combination whereby LGN cells serve as inputs at two sequential processing stages. We further evaluated this model by analysis of pairs of simple cells.

\section{Cell pair analysis}

To investigate the possibility that DS simple cells are derived from a combination of biphasic simple cells and lagged LGN cells, we recorded simultaneously from pairs of potentially connected simple cells, one of which was DS and the other non-DS. These cell pairs had spatially overlapping RFs with similar orientation and spatial frequency tuning properties (see Materials and Methods). An example of such a pair is shown in Figure 6. $A$ and $B$ show the spatiotemporal RFs of the simultaneously recorded DS and non-DS cells, respectively. Assuming that the non-DS cell represents one of the input components to the DS cell, the missing input component (in a two-component linear model) can be obtained by taking the difference of the DS and non-DS RFs. The difference RF (i.e., missing component), shown in Figure 6C, resembles a separable RF plausibly of either LGN or simple-cell origin. In this case, the missing component is slightly lagged relative to the recorded non-DS cell. Because all of our pairs are simple cells, if we find that missing components consistently have relatively long latencies, this implies that late input is derived from lagged LGN cells. However, if we find that non-DS members of cell pairs function as both early and late inputs, this would indicate that direct lagged LGN input is not necessary.

We recorded from 22 pairs of potentially connected cells. Five of the pairs were removed from the analysis because of poor fits (see Materials and Methods). Of the 17 remaining pairs, three have CCHs that indicate monosynaptic input from the non-DS cell to the DS cell. Five pairs show CCHs indicative of common input. For this study, the remainder of the pairs are considered potentially connected in that there may be a connection that is not apparent from the CCH or that the non-DS cell is representative of the temporal response properties available as input to the DS cell.

For each pair, we fit the non-DS RF with a purely separable version of the quadrature fit ( $\alpha=0$; Eq. 10). We then found the best-fitting missing RF, which we also forced to be space-time separable (see Materials and Methods). A depiction of this analysis is shown in Figure 7. The recorded RFs are displayed at the top (Fig. $7 A, B$ ). The fit to the non-DS cell is shown in Figure $7 D$, and the best-fitting missing component in shown in Figure $7 F$. 

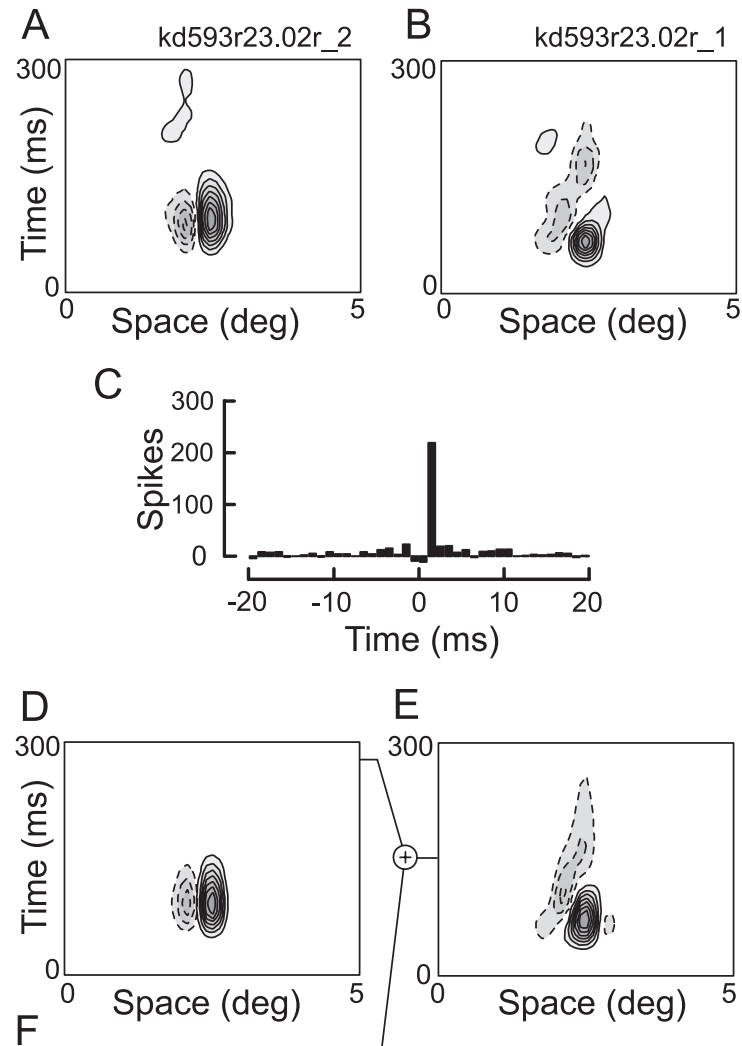

$\mathrm{E}$
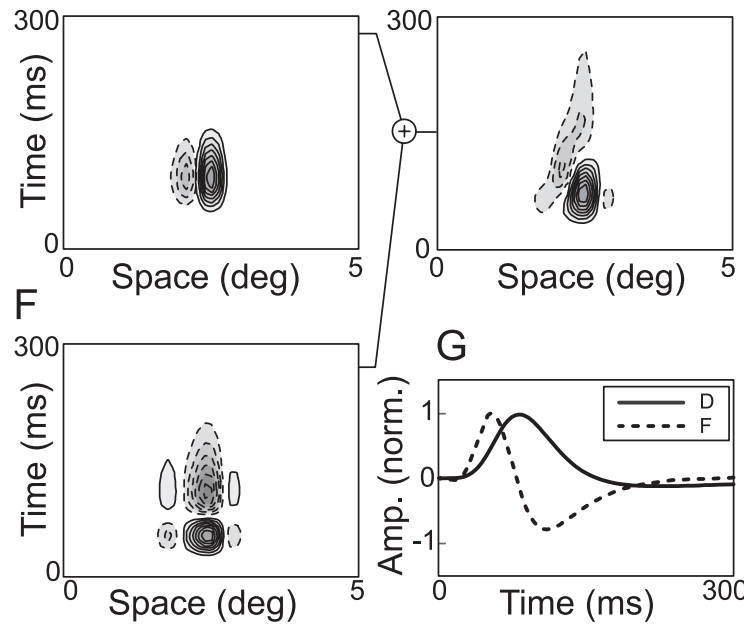

G

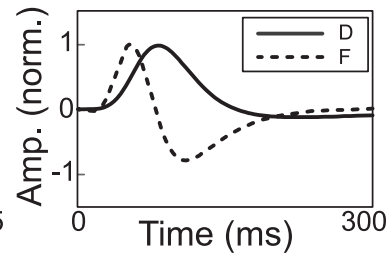

Figure 8. Another cell pair analysis as in Figure 7. For this pair, the $\mathrm{CCH}$ contains a narrow peak offset from zero, which is indicative of a monosynaptic connection from the non-DS to the DS cell. Here the best-fitting missing component $\left(R^{2}=0.85\right)$ produces a temporal phase difference of $-85.2^{\circ}$, which is approximately quadrature. The negative phase value indicates that the non-DS member of the pair functions as the late component. This is an example in which the late component is a simple cell. In other words, direct lagged input is not necessary to produce direction selectivity.

The sum of the non-DS RF and missing RF (Fig. 7E) produces a good fit $\left(R^{2}=0.88\right)$ to the DS RF (Fig. $\left.7 B\right)$. To compare the latencies, the temporal profiles of the non-DS and missing RFs are plotted on the same axes (Fig. 7G) as solid and dashed lines, respectively. Note that the two components are not in temporal quadrature. The missing component is only slightly phase lagged $\left(11^{\circ}\right)$ compared with the recorded non-DS cell. Nevertheless, the small temporal phase difference is enough to produce a good fit to the DS RF. Note that the $\mathrm{CCH}$ for this pair does not exhibit a clear functional connection (Fig. 7C).

Another example of our cell pair analysis is shown in Figure 8. In this case, the $\mathrm{CCH}$ indicates a monosynaptic connection from the non-DS to the DS cell (Fig. $8 C$ ). Here the missing component is in an approximate quadrature relationship with the non-DS member of the pair (Fig. $8 G$, temporal phase difference of $\left.-85.2^{\circ}\right)$. Note that the recorded non-DS cell forms the late input. If lagged LGN afferents always supply the late input, then one would not expect to find pairs of connected simple cells such as this. This is evidence against the need for direct lagged LGN input
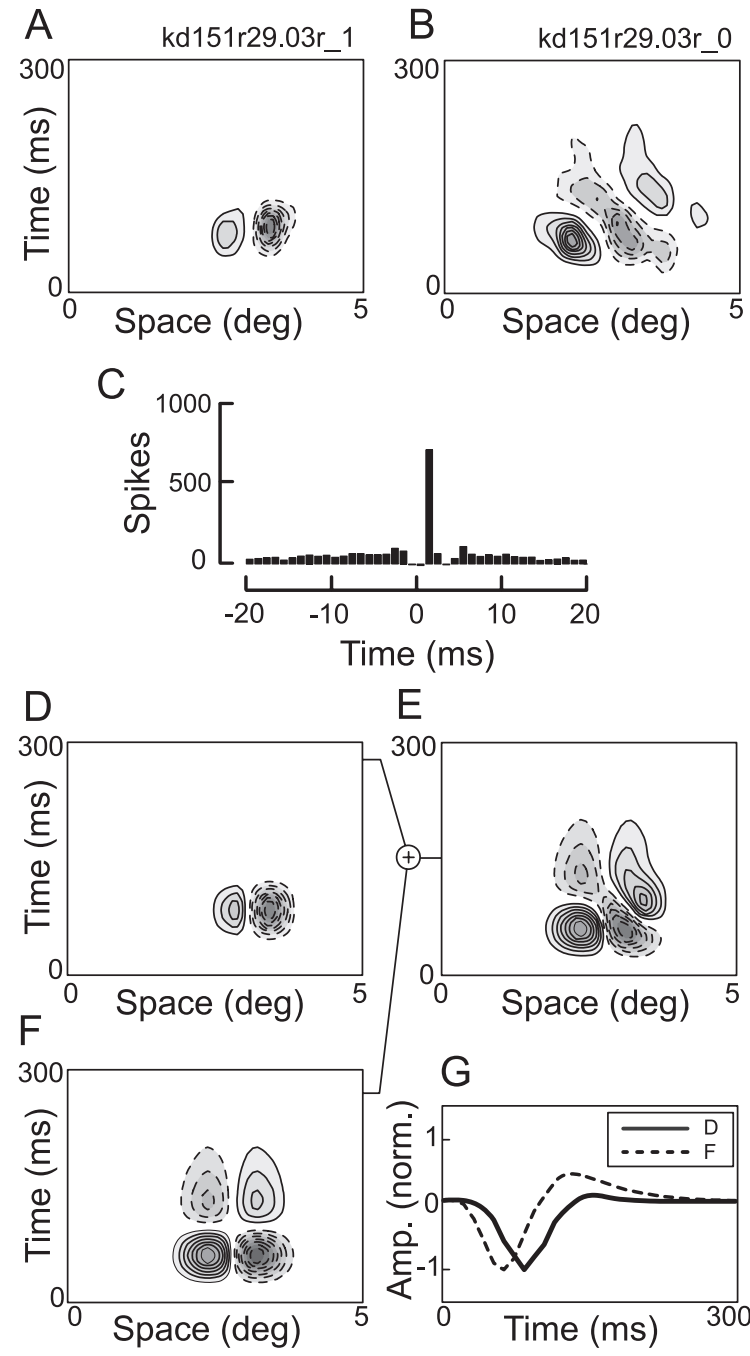

Figure 9. A third example of the cell pair analysis. The layout is the same as in Figure 7. As in Figure 8, this pair exhibits a $\mathrm{CCH}$ indicative of a monosynaptic connection from the DS to the non-DS cell. Here the best-fitting missing component $\left(R^{2}=0.74\right)$ has a temporal phase difference of $-46^{\circ}$, indicating that the measured RF forms the late component with a relationship that is less than quadrature.

in the derivation of direction selectivity. This argument is strengthened by the fact that the $\mathrm{CCH}$ of this pair indicates a monosynaptic functional connection. A second example, also with evidence of a monosynaptic connection, is shown in Figure 9. In this case, the temporal phase difference between the two input components is $-46^{\circ}$. As in the last example, the recorded non-DS simple cell forms the late component.

We find cell pairs with both early and late non-DS input, as indicated by the positive and negative phase differences between the recorded and missing components (Fig. 10A). This suggests that simple cells are forming both the early and the late inputs to DS cells, implying that direct input from lagged LGN cells is not necessary. Also note that the range of phase differences is more or less evenly spread between $-90^{\circ}$ and $+90^{\circ}$. This suggests that temporal quadrature is not required to produce DS cells. These results are consistent with the variable phase model.

\section{Minimum phase fit}

The distributions of latencies shown in Figure 2 are predictions based on quadrature fits to the DS RFs in our sample. For each DS 

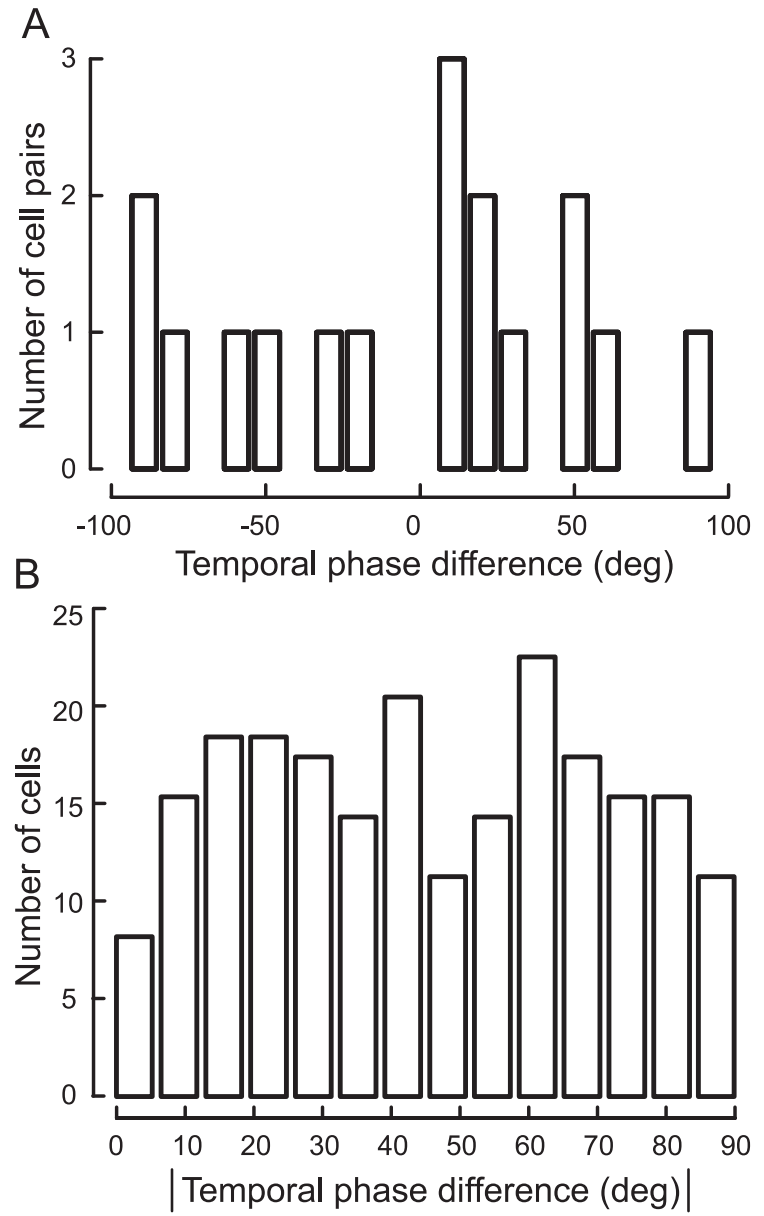

Figure 10. The distribution of temporal phase differences between the two input components. $A$, Phase differences determined by a fitting analysis of cell pairs $(n=17)$. We find both positive and negative phase differences, indicating that non-DS simple cells can form both the early and late input components of DS cells. The phase differences cover the whole range of phases from $-90^{\circ}$ to $+90^{\circ}$, suggesting that DS cells are formed from a pair of inputs with phase differences often less than quadrature. $B, A$ distribution of minimum temporal phase differences needed to fit DS simple cells with the same goodness of fit as for the quadrature model. The distribution is broad, suggesting that temporal quadrature is not necessary for direction selectivity.

RF, we determined the smallest temporal phase difference producing the same goodness of fit as the quadrature model (sequential $F$ test; $p>0.05$ ). The result (Fig. $10 B$ ) is a broad, and relatively flat $\left(p>0.5 ; \chi^{2}\right)$, distribution with only a small proportion $(\sim 7 \%)$ requiring phase differences $>80^{\circ}$. Most DS cells only require temporal phase differences $<55^{\circ}$, suggesting that, although the non-DS latency distributions do not meet the requirements for quadrature inputs, they might be sufficient to supply the minimum phase differences needed to produce DS simple cells.

When the temporal phase difference of two input cells is $<90^{\circ}$, there is not a clear separation between monophasic and biphasic profiles. However, if we define the component with the higher biphasic index as the biphasic component and the other as the monophasic component, we can compare the latency distributions obtained through the quadrature and variable phase models. Figure 11 shows the minimum phase latency distributions for monophasic-like (solid line) and biphasic-like (short and long dashed lines) components. Because the minimum required temporal phase differences are typically much less than $90^{\circ}$, the latency distribution of the more monophasic of the two components greatly overlaps the latency distribution of the first peak of

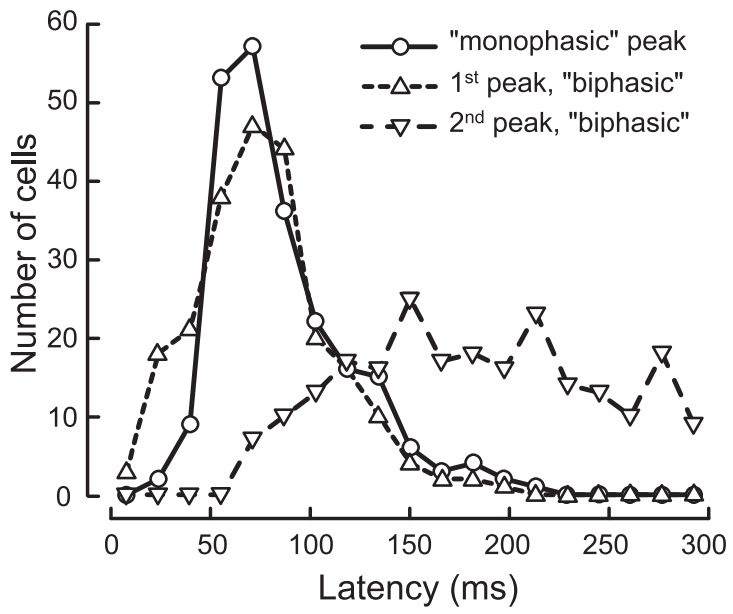

Figure 11. Analysis of the minimum temporal phase necessary to fit DS cells shows a broad distribution with very few cells requiring quadrature. This figure shows the distribution of minimum phase differences in terms of latency-to-peak. Solid and dashed lines represent the distribution of peak latencies for the more monophasic (solid line; mean, $85.9 \pm 34.6 \mathrm{msec}$ ) and more biphasic (short and long dashed lines; means, $76.6 \pm 33.9$ and $183.0 \pm 61.1 \mathrm{msec}$, respectively) of the two components of the variable phase fit, respectively. The more monophasic component is defined as the one with the smaller biphasic index.

the more biphasic of the components. Both are within the range of first peak latencies for non-DS simple cells (Fig. 4). This indicates that the temporal characteristics of non-DS simple cells are sufficiently diverse to generate the linear stage of DS simple cells found in the primary visual cortex of the cat.

Considered together, these results suggest that the directionally selective responses of simple cells are derived by linear mechanisms and enhanced by static nonlinearities. The linear stage receives input from two or more non-DS simple cells with spatial and temporal phase offsets. Unlike the quadrature model, these phase offsets are not constrained to be a quarter of a cycle, and a wide range of temporal phase offsets are observed.

\section{Discussion}

We sought to determine the neural circuitry underlying the generation of DS simple cells in the primary visual cortex. We considered plausible circuits whereby the linear DS RFs are formed from the sum of non-DS inputs with spatial and temporal phase differences. The first neural circuit we considered is based on the first stage of the energy model and specifies spatial and temporal phase differences in quadrature (Adelson and Bergen, 1985; Watson and Ahumada, 1985). In this circuit, different degrees of directional selectivity are obtained by summing the inputs with different relative weights. This circuit does not adequately account for our data. Our sample of non-DS simple cells comprises mostly short to moderate latencies and lacks many RFs with a quarter-cycle temporal phase offset. This implies a possible role for direct input from lagged LGN cells; however, our analysis of pairs of simple cells reveals that non-DS simple cells can provide input for both the early- and late-phase components. This suggests that direct input from lagged LGN cells is not necessary.

The second circuit we considered combines inputs with equal weights and produces different degrees of directional selectivity by allowing the phase differences to vary. Our results are consistent with this circuit. We find that the minimum temporal phase required to form DS simple cells is typically much less than quadrature. Furthermore, the distribution of minimum phases agrees with the latency distributions of non-DS simple cells. Con- 
sidered together, our results suggest that the linear RFs of DS simple cells are derived from the sum of two non-DS simple cells with temporal phase differences that are often less than quadrature. Relatively small temporal phase differences, when combined with static nonlinearities, can result in highly directionselective response properties.

\section{Distribution of direction selectivity}

In this study, we classified $74 \%$ of simple cells in area 17 as directionally selective. This value is on the high end of the range of percentages reported for the cat. Using the same criteria (DSI $>0.5$ ), most studies report somewhere between 60 and 70\% DS cells (Hamilton et al., 1989; Gizzi et al., 1990; McLean et al., 1994; Carandini and Ferster, 2000). A couple of studies suggest that fewer than 50\% of simple cells are DS (Orban et al., 1981; Berman et al., 1987), and some find up to $80 \%$ (Humphrey and Saul, 1998). The relatively wide range of reported percentages can be accounted for, in part, by variations in stimulus parameters (Hammond and Pomfrett, 1990; Casanova et al., 1992) and possibly different electrode penetrations (Berman et al., 1987). The percentages reported in the cat are typically higher than in the monkey, which range from 20 to 60\% (Schiller et al., 1976; De Valois et al., 1982, 2000; Albright, 1984; Hamilton et al., 1989; O'Keefe et al., 1998). Some studies in the cat find a trimodal distribution of DSI values with peaks near $0,0.5$, and 1 (Berman et al., 1987). This is similar to what is observed in the monkey (De Valois et al., 2000). However, we find a more continuous distribution of DSI values (Fig. 1). This difference in distributions, together with the relatively high percentage of DS cells, indicates that our sample might under represent non-DS cells. One possibility is that our sample lacks many long-latency non-DS simple cells just as we find relatively few lagged LGN cells. However, we have no direct evidence regarding this possibility.

\section{Multiple derivations of directional selectivity}

The results presented here suggest that direct input from lagged LGN cells is not necessary to produce the range of DS simple cells observed in area 17 . We find evidence for non-DS simple cell input for both early- and late-phase components. However, this does not imply that lagged cells are not involved. Late-phase non-DS simple cells might receive mainly lagged input, and some DS cells may be generated by direct lagged input whereas others receive mainly non-DS simple cell input. Evidence that lagged cells feed into simple cells (Alonso et al., 2001) and that cooling the visual cortex does not diminish directional selectivity for at least some cells (Ferster et al., 1996) suggests that lagged cells are involved at some level. However, area 18 appears to have a higher percentage of DS cells (Orban et al., 1981; McLean et al., 1994), and it receives mainly Y LGN input (Humphrey et al., 1985), yet it is reported that $\mathrm{Y}$ cells are almost entirely nonlagged (Saul and Humphrey, 1990; Mastronarde et al., 1991). This raises the possibility that some DS simple cells are generated mainly by the spatial and temporal phase offsets of direct LGN afferents and that others, such as the cell pairs with monosynaptic connections reported here, and possibly area 18 cells, are generated mainly from non-DS simple cells.

The model proposed here, in which non-DS simple cells sum together to form DS simple cells, is similar to that proposed for the monkey (De Valois et al., 2000) and accounts for some, but not all, of the properties reported for DS cells. For instance, there is evidence that directional selectivity is dependent on intracortical inhibitory mechanisms (Tsumoto et al., 1979; Sillito, 1984; Livingstone, 1998). Perhaps the blockage of inhibitory mecha- nisms reduces directional selectivity by affecting the push-pull mechanisms of simple cells (Hubel and Wiesel, 1962; Palmer and Davis, 1981; Ferster, 1988; Hirsch et al., 1998). Another possibility is that some DS cells might be generated from the difference instead of the sum of two non-DS inputs. Theoretically, subtractive mechanisms can be as effective as additive ones in the generation of space-time inseparable RFs. Although we did not find direct evidence for inhibitory connections from non-DS to DS simple cells, these connections are difficult to detect using extracellular techniques (Aertsen and Gerstein, 1985; Melssen and Epping, 1987).

Some DS simple cells exhibit almost completely separable space-time RFs but nevertheless are DS when tested with drifting gratings. We find a small number of such cells in this study but treat them as non-DS cells for the purpose of analyzing their RFs. Other studies suggest that these cells are mostly found in layer 6 and exhibit different properties than DS simple cells found in other layers (Murthy et al., 1998). Therefore, it appears that there may be several circuits that generate DS simple cells in the visual cortex.

\section{Comparison with findings in the primate}

In recent studies of simple cells in an anesthetized monkey preparation, populations are reported of monophasic and biphasic non-DS RFs with timing characteristics that agree with a quadrature model of direction selectivity (De Valois and Cottaris, 1998; De Valois et al., 2000; Conway and Livingstone, 2003). This is interpreted as evidence for a hierarchical system in which LGN cells feed into non-DS simple cells, which in turn combine to generate DS simple cells. The data reported here, from a cat preparation, also are consistent with a hierarchical system but not necessarily with quadrature phase differences. In the cat, it appears that smaller temporal phase differences are involved, which may be accentuated by higher output nonlinearities.

\section{References}

Adelson EH, Bergen JR (1985) Spatiotemporal energy models for the perception of motion. J Opt Soc Am A 2:284-299.

Aertsen AM, Gerstein GL (1985) Evaluation of neuronal connectivity: sensitivity of cross-correlation. Brain Res 340:341-354.

Albright TD (1984) Direction and orientation selectivity of neurons in visual area MT of the macaque. J Neurophysiol 52:1106-1130.

Alonso JM, Usrey WM, Reid RC (2001) Rules of connectivity between geniculate cells and simple cells in cat primary visual cortex. J Neurosci 21:4002-4015.

Anzai A, Ohzawa I, Freeman RD (1999) Neural mechanisms for processing binocular information. I. Simple cells. J Neurophysiol 82:891-908.

Barlow HB, Levick WR (1965) The mechanism of directionally selective units in rabbit's retina. J Physiol (Lond) 178:477-504.

Berman NE, Wilkes ME, Payne BR (1987) Organization of orientation and direction selectivity in areas 17 and 18 of cat cerebral cortex. J Neurophysiol 58:676-699.

Borg-Graham LJ (2001) The computation of directional selectivity in the retina occurs presynaptic to the ganglion cell. Nat Neurosci 4:176-183.

Brody CD (1998) Slow covariations in neuronal resting potentials can lead to artefactually fast cross-correlations in their spike trains. J Neurophysiol 80:3345-3351.

Brody CD (1999) Correlations without synchrony. Neural Comput 11:1537-1551.

Cai D, DeAngelis GC, Freeman RD (1997) Spatiotemporal receptive field organization in the lateral geniculate nucleus of cats and kittens. J Neurophysiol 78:1045-1061.

Carandini M, Ferster D (2000) Membrane potential and firing rate in cat primary visual cortex. J Neurosci 20:470-484.

Casanova C, Nordmann JP, Ohzawa I, Freeman RD (1992) Direction selectivity of cells in the cat's striate cortex: differences between bar and grating stimuli. Vis Neurosci 9:505-513. 
Cleland BG, Levick WR, Morstyn R, Wagner HG (1976) Lateral geniculate relay of slowly conducting retinal afferents to cat visual cortex. J Physiol (Lond) 255:299-320.

Conway BR, Livingstone MS (2003) Space-time maps and two-bar interactions of different classes of direction-selective cells in macaque V-1. J Neurophysiol 89:2726-2742.

De Valois RL, Cottaris NP (1998) Inputs to directionally selective simple cells in macaque striate cortex. Proc Natl Acad Sci USA 95:14488-14493.

De Valois RL, Yund EW, Hepler N (1982) The orientation and direction selectivity of cells in macaque visual cortex. Vision Res 22:531-544.

De Valois RL, Cottaris NP, Mahon LE, Elfar SD, Wilson JA (2000) Spatial and temporal receptive fields of geniculate and cortical cells and directional selectivity. Vision Res 40:3685-3702.

Dean AF, Tolhurst DJ (1986) Factors influencing the temporal phase of response to bar and grating stimuli for simple cells in the cat striate cortex. Exp Brain Res 62:143-151.

DeAngelis GC, Ohzawa I, Freeman RD (1993) Spatiotemporal organization of simple-cell receptive fields in the cat's striate cortex. II. Linearity of temporal and spatial summation. J Neurophysiol 69:1118-1135.

DeAngelis GC, Ghose GM, Ohzawa I, Freeman RD (1999) Functional micro-organization of primary visual cortex: receptive field analysis of nearby neurons. J Neurosci 19:4046-4064.

Ferster D (1988) Spatially opponent excitation and inhibition in simple cells of the cat visual cortex. J Neurosci 8:1172-1180.

Ferster D, Chung S, Wheat H (1996) Orientation selectivity of thalamic input to simple cells of cat visual cortex. Nature 380:249-252.

Field DJ, Tolhurst DJ (1986) The structure and symmetry of simple-cell receptive-field profiles in the cat's visual cortex. Proc R Soc Lond B Biol Sci 228:379-400.

Freeman RD, Ohzawa I (1990) On the neurophysiological organization of binocular vision. Vision Res 30:1661-1676.

Gabor D (1946) Theory of communication. J Inst Electr Eng 93:429-457.

Gerstein GL, Perkel DH (1969) Simultaneously recorded trains of action potentials: analysis and functional interpretation. Science 164:828-830.

Ghose GM, Freeman RD, Ohzawa I (1994) Local intracortical connections in the cat's visual cortex: postnatal development and plasticity. J Neurophysiol 72:1290-1303.

Gizzi MS, Katz E, Schumer RA, Movshon JA (1990) Selectivity for orientation and direction of motion of single neurons in cat striate and extrastriate visual cortex. J Neurophysiol 63:1529-1543.

Hamilton DB, Albrecht DG, Geisler WS (1989) Visual cortical receptive fields in monkey and cat: spatial and temporal phase transfer function. Vision Res 29:1285-1308.

Hammond P, Pomfrett CJ (1990) Influence of spatial frequency on tuning and bias for orientation and direction in the cat's striate cortex. Vision Res 30:359-369.

Hawken MJ, Shapley RM, Grosof DH (1996) Temporal-frequency selectivity in monkey visual cortex. Vis Neurosci 13:477-492.

Hirsch JA, Alonso JM, Reid RC, Martinez LM (1998) Synaptic integration in striate cortical simple cells. J Neurosci 18:9517-9528.

Horsley V, Clarke R (1908) The structure and functions of the cerebellum examined by a new method. Brain 31:45-124.

Hubel DH, Wiesel TN (1962) Receptive fields, binocular interaction and functional architecture in the cat's visual cortex. J Physiol (Lond) 160:106-154.

Humphrey AL, Saul AB (1998) Strobe rearing reduces direction selectivity in area 17 by altering spatiotemporal receptive-field structure. J Neurophysiol 80:2991-3004.

Humphrey AL, Weller RE (1988) Functionally distinct groups of X-cells in the lateral geniculate nucleus of the cat. J Comp Neurol 268:429-447.

Humphrey AL, Sur M, Uhlrich DJ, Sherman SM (1985) Termination patterns of individual $\mathrm{X}$ - and $\mathrm{Y}$-cell axons in the visual cortex of the cat: projections to area 18 , to the $17 / 18$ border region, and to both areas 17 and 18. J Comp Neurol 233:190-212.

Jagadeesh B, Wheat HS, Kontsevich LL, Tyler CW, Ferster D (1997) Direction selectivity of synaptic potentials in simple cells of the cat visual cortex. J Neurophysiol 78:2772-2789.

Jones JP, Palmer LA (1987) The two-dimensional spatial structure of simple receptive fields in cat striate cortex. J Neurophysiol 58:1187-1211.
Kwon YH, Nelson SB, Toth LJ, Sur M (1992) Effect of stimulus contrast and size on NMDA receptor activity in cat lateral geniculate nucleus. J Neurophysiol 68:182-196.

Levick WR (1972) Another tungsten microelectrode. Med Biol Eng 10:510-515.

Livingstone MS (1998) Mechanisms of direction selectivity in macaque V1. Neuron 20:509-526.

Mastronarde DN (1987) Two classes of single-input X-cells in cat lateral geniculate nucleus. II. Retinal inputs and the generation of receptive-field properties. J Neurophysiol 57:381-413.

Mastronarde DN, Humphrey AL, Saul AB (1991) Lagged Y cells in the cat lateral geniculate nucleus. Vis Neurosci 7:191-200.

McLean J, Raab S, Palmer LA (1994) Contribution of linear mechanisms to the specification of local motion by simple cells in areas 17 and 18 of the cat. Vis Neurosci 11:271-294.

Mechler F, Ringach DL (2002) On the classification of simple and complex cells. Vision Res 42:1017-1033.

Melssen WJ, Epping WJ (1987) Detection and estimation of neural connectivity based on crosscorrelation analysis. Biol Cybern 57:403-414.

Menz MD, Freeman RD (2004) Functional connectivity of disparity tuned neurons in the visual cortex. J Neurophysiol 91:1794-1807.

Murthy A, Humphrey AL, Saul AB, Feidler JC (1998) Laminar differences in the spatiotemporal structure of simple cell receptive fields in cat area 17. Vis Neurosci 15:239-256.

Nishimoto S, DeAngelis GC, Ohzawa I, Freeman RD (2001) Organization of spatiotemporal receptive field of direction-selective simple cells in the cat visual cortex. Neuroscience Res Suppl 25:PA2-PA116.

O'Keefe LP, Levitt JB, Kiper DC, Shapley RM, Movshon JA (1998) Functional organization of owl monkey lateral geniculate nucleus and visual cortex. J Neurophysiol 80:594-609.

Orban GA, Kennedy H, Maes H (1981) Response to movement of neurons in areas 17 and 18 of the cat: direction selectivity. J Neurophysiol 45:1059-1073.

Palmer LA, Davis TL (1981) Receptive-field structure in cat striate cortex. J Neurophysiol 46:260-276.

Perkel DH, Gerstein GL, Moore GP (1967) Neuronal spike trains and stochastic point processes. II. Simultaneous spike trains. Biophys J 7:419-440.

Saul AB, Humphrey AL (1990) Spatial and temporal response properties of lagged and nonlagged cells in cat lateral geniculate nucleus. J Neurophysiol 64:206-224.

Saul AB, Humphrey AL (1992) Evidence of input from lagged cells in the lateral geniculate nucleus to simple cells in cortical area 17 of the cat. J Neurophysiol 68:1190-1208.

Schiller PH, Finlay BL, Volman SF (1976) Quantitative studies of single-cell properties in monkey striate cortex. I. Spatiotemporal organization of receptive fields. J Neurophysiol 39:1288-1319.

Sillito AM (1984) Functional considerations of the operation of GABAergic inhibitory process in the visual cortex. In: Cerebral cortex, pp 91-117. New York: Plenum.

Skottun BC, De Valois RL, Grosof DH, Movshon JA, Albrecht DG, Bonds AB (1991) Classifying simple and complex cells on the basis of response modulation. Vision Res 31:1079-1086.

Taylor WR, Vaney DI (2003) New directions in retinal research. Trends Neurosci 26:379-385.

Toyama K, Kimura M, Tanaka K (1981) Cross-correlation analysis of interneuronal connectivity in cat visual cortex. J Neurophysiol 46:191-201.

Tsumoto T, Eckart W, Creutzfeldt OD (1979) Modification of orientation sensitivity of cat visual cortex neurons by removal of GABA-mediated inhibition. Exp Brain Res 34:351-363.

Watson AB, Ahumada Jr AJ (1985) Model of human visual-motion sensing. J Opt Soc Am A 2:322-341.

Wilson PD, Rowe MH, Stone J (1976) Properties of relay cells in cat's lateral geniculate nucleus: a comparison of W-cells with X- and Y-cells. J Neurophysiol 39:1193-1209.

Wolfe J, Palmer LA (1998) Temporal diversity in the lateral geniculate nucleus of cat. Vis Neurosci 15:653-675. 